\title{
Application of Trial Equation Method for Solving the Getmanou Equation
}

\author{
Li Yang \\ Department of Mathematics, Northeast Petroleum University, Daqing, China \\ Email: liyang120918@163.com \\ Received 21 March 2014; revised 21 April 2014; accepted 28 April 2014 \\ Copyright (C) 2014 by author and Scientific Research Publishing Inc. \\ This work is licensed under the Creative Commons Attribution International License (CC BY). \\ http://creativecommons.org/licenses/by/4.0/ \\ (c) (i) Open Access
}

\begin{abstract}
Under the travelling wave transformation, some nonlinear partial differential equations such as the Getmanou equation are transformed to ordinary differential equation. Then using trial equation method and combing complete discrimination system for polynomial, the classifications of all single traveling wave solution to this equation are obtained.
\end{abstract}

\section{Keywords}

The Nonlinear Partial Differential Equation, Trial Equation Method, Complete Discrimination System for Polynomia, Traveling Wave Transform, the Getmanou Equation

\section{Introduction}

Many problems in natural and engineering sciences are modeled by partial differential equations (PDE). Looking for the solutions of the equation, especially the exact solutions, is very important. These exact solutions can describe many important phenomena in physics and other fields and also help physicists to understand the mechanisms of the complicated physical phenomena. Many mathematicians and physicists work in the field, and a variety of powerful methods have been employed to study nonlinear phenomena, such as the inverse scattering transform [1], the Bäcklund transformation method [2], the Darboux transformation [3], the homogeneous balance method [4], the tanh function method [5], the exp-function method [6], the G'/G-expansion method [7], and so on.

Recently, Professor Liu proposed a powerful method named trial equation method [8] [9] for finding exact solutions to nonlinear differential equations. In this paper, I mainly use Liu's trial equation method and the theory of complete discrimination system for the fouth-order polynomial [10]-[12] to solve exact solutions of the Getmanou equation which has already been solved based on discrimination system of the fifth-order polynomial by Fan [13]. But as we can see, the solving process is very simple and clear with the trial equation method 
combined with complete discrimination system for polynomial.

\section{Application of Trial Equation Method}

The Getmanou equation [12] reads as

$$
u_{x t}+\frac{u_{x} u_{t}}{1-u^{2}}-u\left(1-u^{2}\right)=0
$$

Or equivalently

$$
\left(1-u^{2}\right) u_{x t}+u_{x} u_{t}-u\left(1-u^{2}\right)^{2}=0 .
$$

Taking the traveling wave transformation $u=u\left(\xi_{1}\right)$ and $\xi_{1}=k x+\omega t$, we can obtain the corresponding ODE

$$
k \omega\left(1-u^{2}\right) u^{\prime \prime}+k \omega\left(u^{\prime}\right)^{2}-u^{5}+2 u^{3}-u=0 .
$$

we take the trial equation as follows

$$
u^{\prime \prime}=a_{0}+a_{1} u+\cdots+a_{m} u^{m} .
$$

According to the trial equation method of rank homogeneous equation, balancing $u^{2} u^{\prime \prime}$ with $u^{5}$ gets $m=3$.

Equation (4) has the following specific form

$$
u^{\prime \prime}=a_{3} u^{3}+a_{2} u^{2}+a_{1} u+a_{0} .
$$

From Equation (5), we get

$$
\left(u^{\prime}\right)^{2}=\frac{1}{2} a_{3} u^{4}+\frac{2}{3} a_{2} u^{3}+a_{1} u^{2}+2 a_{0} u+d .
$$

Substituting Equations (5) and (6) into Equation (3), we have

$$
r_{5} u^{5}+r_{4} u^{4}+r_{3} u^{3}+r_{2} u^{2}+r_{1} u+r_{0}=0 .
$$

where

$$
\begin{aligned}
& r_{0}=k \omega a_{0}+k \omega d . \\
& r_{1}=k \omega a_{1}+2 k \omega a_{0}-1 . \\
& r_{2}=k \omega a_{2}+k \omega a_{1}-k \omega a_{0} . \\
& r_{3}=k \omega a_{3}+\frac{2}{3} k \omega a_{2}-k \omega a_{1}+2 . \\
& r_{4}=\frac{1}{2} k \omega a_{3}-k \omega a_{2} . \\
& r_{5}=-k \omega a_{3}-1 .
\end{aligned}
$$

Let the coefficient $r_{i}(i=0,1,2,3,4,5)$ be zero, we will yield nonlinear algebraic equations. Solving the equations, we will determine the values of $a_{0}, a_{1}, a_{2}, a_{3}, d$. We get

$$
a_{0}=\frac{1}{6 k \omega}, a_{1}=\frac{2}{3 k \omega}, a_{2}=-\frac{1}{2 k \omega}, a_{3}=-\frac{1}{k \omega}, d=\frac{1}{6 k \omega} .
$$

When $a_{3}>0$, we take transformations as follows

$$
w=\left(\frac{1}{2} a_{3}\right)^{\frac{1}{4}}\left(u+\frac{a_{2}}{3 a_{3}}\right), \xi=\left(\frac{1}{2} a_{3}\right)^{\frac{1}{4}} \xi_{1} .
$$

Then Equation (6) becomes

$$
\left(w^{\prime}\right)^{2}=w^{4}+p w^{2}+q w+r .
$$


where $w$ is a function of $\xi$ and

$$
\begin{aligned}
& p=a_{1}\left(\frac{1}{2} a_{3}\right)^{-\frac{1}{2}}-\frac{1}{3}\left(\frac{1}{2} a_{3}\right)^{-\frac{1}{2}} a_{2}^{2} a_{3}^{-1} . \\
& q=-\frac{2}{3} a_{1}\left(\frac{1}{2} a_{3}\right)^{-\frac{1}{4}} a_{2} a_{3}^{-1}+\frac{4}{27}\left(\frac{1}{2} a_{3}\right)^{-\frac{1}{4}} a_{2}^{3} a_{3}^{-2}+2 a_{0}\left(\frac{1}{2} a_{3}\right)^{-\frac{1}{4}} . \\
& r=a_{0}-\frac{2}{3} a_{0} a_{2} a_{3}^{-1}+\frac{1}{9} a_{1} a_{2}^{2} a_{3}^{-2}-\frac{1}{54} a_{2}^{4} a_{3}^{-3} .
\end{aligned}
$$

When $a_{3}<0$, we take transformations as follows

$$
w=\left(-\frac{1}{2} a_{3}\right)^{\frac{1}{4}}\left(u+\frac{a_{2}}{3 a_{3}}\right), \xi=\left(-\frac{1}{2} a_{3}\right)^{\frac{1}{4}} \xi_{1} .
$$

Then Equation (6) becomes

$$
\left(w^{\prime}\right)^{2}=-\left(w^{4}+p w^{2}+q w+r\right) .
$$

where $w$ is a function of $\xi$ and

$$
\begin{aligned}
& p=-a_{1}\left(-\frac{1}{2} a_{3}\right)^{-\frac{1}{2}}+\frac{1}{3}\left(-\frac{1}{2} a_{3}\right)^{-\frac{1}{2}} a_{2}^{2} a_{3}^{-1} . \\
& q=\frac{2}{3} a_{1}\left(-\frac{1}{2} a_{3}\right)^{-\frac{1}{4}} a_{2} a_{3}^{-1}-\frac{4}{27}\left(-\frac{1}{2} a_{3}\right)^{-\frac{1}{4}} a_{2}^{3} a_{3}^{-2}-2 a_{0}\left(-\frac{1}{2} a_{3}\right)^{-\frac{1}{4}} . \\
& r=-a_{0}+\frac{2}{3} a_{0} a_{2} a_{3}^{-1}-\frac{1}{9} a_{1} a_{2}^{2} a_{3}^{-2}+\frac{1}{54} a_{2}^{4} a_{3}^{-3} .
\end{aligned}
$$

We write the complete discrimination system for polynomial $F(w)=w^{4}+p w^{2}+q w+r$ as follows $D_{1}=4, D_{2}=-p, D_{3}=8 r p-2 p^{3}-9 q^{2}, D_{4}=4 p^{4} r-p^{3} q^{2}+36 p r q^{2}-32 r^{2} p^{2}-\frac{27}{4} q^{4}+64 r^{3}, E_{2}=9 q^{2}-32 p r$.

Then we consider the following ODE

$$
\left(w^{\prime}\right)^{2}=\varepsilon\left(w^{4}+p w^{2}+q w+r\right) .
$$

where $\varepsilon= \pm 1$. Rewrite Equation (17) by integral form as follows

$$
\pm\left(\xi-\xi_{0}\right)=\int \frac{\mathrm{d} w}{\sqrt{\varepsilon\left(w^{4}+p w^{2}+q w+r\right)}} .
$$

\section{Classification of the Traveling Wave Solutions}

According to the complete discrimination system for the fouth order polynomial, we give the corresponding single traveling wave solutions to Equation (1).

Case 1. $D_{4}=0, D_{3}=0, D_{2}<0$. Then we have

$$
F(w)=\left[\left(w-l_{1}\right)^{2}+s_{1}^{2}\right]^{2} .
$$

where $l_{1}$ and $s_{1}$ are real numbers, $s_{1}>0$.

When $\varepsilon=1$, we have

$$
w=s_{1} \tan \left[s_{1}\left(\xi-\xi_{0}\right)\right]+l_{1} .
$$

The corresponding solution is 


$$
u=\left(\frac{1}{2} a_{3}\right)^{-\frac{1}{4}}\left\{s_{1} \tan \left[s_{1}\left(\frac{1}{2} a_{3}\right)^{\frac{1}{4}}\left(\xi_{1}-\xi_{0}\right)\right]+l_{1}\right\}-\frac{a_{2}}{3 a_{3}} .
$$

Case 2. $D_{4}=0, D_{3}=0, D_{2}=0$. Then we have

$$
F(w)=w^{4} .
$$

When $\varepsilon=1$, we have

$$
w=-\frac{1}{\left(\xi-\xi_{0}\right)}
$$

The corresponding solution is

$$
u=-\left(\frac{1}{2} a_{3}\right)^{-\frac{1}{2}} \frac{1}{\left(\xi_{1}-\xi_{0}\right)}-\frac{a_{2}}{3 a_{3}} .
$$

Case 3. $D_{4}=0, D_{3}=0, D_{2}>0, E_{2}=0$. Then we have

$$
F(w)=(w-\alpha)^{2}(w-\beta)^{2} .
$$

where $\alpha$ and $\beta$ are real numbers, $\alpha>\beta$.

When $\varepsilon=1$

(i) If $w>\alpha$ or $w<\beta$, we have

$$
w=\frac{\beta-\alpha}{2}\left[\operatorname{coth} \frac{\alpha-\beta}{2}\left(\xi-\xi_{0}\right)-1\right]+\beta .
$$

The corresponding solution is

$$
u=\left(\frac{1}{2} a_{3}\right)^{-\frac{1}{4}}\left\{\frac{\beta-\alpha}{2}\left[\operatorname{coth} \frac{\alpha-\beta}{2}\left(\frac{1}{2} a_{3}\right)^{\frac{1}{4}}\left(\xi_{1}-\xi_{0}\right)-1\right]+\beta\right\}-\frac{a_{2}}{3 a_{3}} .
$$

(ii) If $\alpha>w>\beta$, we have

$$
w=\frac{\beta-\alpha}{2}\left[\tanh \frac{\alpha-\beta}{2}\left(\xi-\xi_{0}\right)-1\right]+\beta .
$$

The corresponding solution is

$$
u=\left(\frac{1}{2} a_{3}\right)^{-\frac{1}{4}}\left\{\frac{\beta-\alpha}{2}\left[\tanh \frac{\alpha-\beta}{2}\left(\frac{1}{2} a_{3}\right)^{\frac{1}{4}}\left(\xi_{1}-\xi_{0}\right)-1\right]+\beta\right\}-\frac{a_{2}}{3 a_{3}} .
$$

Case 4. $D_{4}=0, D_{3}>0, D_{2}>0$. Then we have

$$
F(w)=(w-\alpha)^{2}(w-\beta)(w-\gamma) .
$$

where $\alpha, \beta, \gamma$ are real numbers, and $\beta>\gamma$.

When $\varepsilon=1$

(i) If $\alpha>\beta, w>\beta$ or if $\alpha<\gamma, w<\gamma$, we have

$$
\pm\left(\xi-\xi_{0}\right)=\frac{1}{\sqrt{(\alpha-\beta)(\alpha-\gamma)}} \ln \frac{[\sqrt{(w-\beta)(\alpha-\gamma)}-\sqrt{(\alpha-\beta)(w-\gamma)}]^{2}}{|w-\alpha|} \text {. }
$$

The corresponding solution is 


$$
\begin{aligned}
& \pm\left(\frac{1}{2} a_{3}\right)^{\frac{1}{4}}\left(\xi_{1}-\xi_{0}\right) \\
& =\frac{1}{\sqrt{(\alpha-\beta)(\alpha-\gamma)}} \ln \frac{\left\{\sqrt{\left[\left(\frac{1}{2} a_{3}\right)^{\frac{1}{4}}\left(u+\frac{a_{2}}{3 a_{3}}\right)-\beta\right](\alpha-\gamma)}-\sqrt{(\alpha-\beta)\left[\left(\frac{1}{2} a_{3}\right)^{\frac{1}{4}}\left(u+\frac{a_{2}}{3 a_{3}}\right)-\gamma\right]}\right\}^{2}}{\left|\left(\frac{1}{2} a_{3}\right)^{\frac{1}{4}}\left(u+\frac{a_{2}}{3 a_{3}}\right)-\alpha\right|} .
\end{aligned}
$$

(ii) If $\alpha>\beta, w<\gamma$ or if $\alpha<\gamma, w<\beta$, we have

$$
\pm\left(\xi-\xi_{0}\right)=\frac{1}{\sqrt{(\alpha-\beta)(\alpha-\gamma)}} \ln \frac{[\sqrt{(w-\beta)(\gamma-\alpha)}-\sqrt{(\beta-\alpha)(w-\gamma)}]^{2}}{|w-\alpha|} .
$$

The corresponding solution is

$$
\begin{aligned}
& \pm\left(\frac{1}{2} a_{3}\right)^{\frac{1}{4}}\left(\xi_{1}-\xi_{0}\right) \\
& =\frac{\left\{\sqrt{\left[\left(\frac{1}{2} a_{3}\right)^{\frac{1}{4}}\left(u+\frac{a_{2}}{3 a_{3}}\right)-\beta\right](\gamma-\alpha)}-\sqrt{\left.(\beta-\alpha)\left[\left(\frac{1}{2} a_{3}\right)^{\frac{1}{4}}\left(u+\frac{a_{2}}{3 a_{3}}\right)-\gamma\right]\right\}^{2}}\right.}{\sqrt{(\alpha-\beta)(\alpha-\gamma)}} \ln \frac{\left\{\left(\frac{1}{2} a_{3}\right)^{\frac{1}{4}}\left(u+\frac{a_{2}}{3 a_{3}}\right)-\alpha \mid\right.}{|(\alpha)|} .
\end{aligned}
$$

(iii) If $\beta>\alpha>\gamma$, we have

$$
\pm\left(\xi-\xi_{0}\right)=\frac{1}{\sqrt{(\beta-\alpha)(\alpha-\gamma)}} \arcsin \frac{(w-\beta)(\alpha-\gamma)+(\alpha-\beta)(w-\gamma)}{|(w-\alpha)(\beta-\gamma)|} .
$$

The corresponding solution is

$$
\begin{aligned}
& \pm\left(\frac{1}{2} a_{3}\right)^{\frac{1}{4}}\left(\xi_{1}-\xi_{0}\right) \\
& =\frac{1}{\sqrt{(\beta-\alpha)(\alpha-\gamma)}} \arcsin \frac{\left[\left(\frac{1}{2} a_{3}\right)^{\frac{1}{4}}\left(u+\frac{a_{2}}{3 a_{3}}\right)-\beta\right](\alpha-\gamma)+(\alpha-\beta)\left[\left(\frac{1}{2} a_{3}\right)^{\frac{1}{4}}\left(u+\frac{a_{2}}{3 a_{3}}\right)-\gamma\right]}{\left|\left[\left(\frac{1}{2} a_{3}\right)^{\frac{1}{4}}\left(u+\frac{a_{2}}{3 a_{3}}\right)-\alpha\right](\beta-\gamma)\right|} .
\end{aligned}
$$

When $\varepsilon=-1$

(i) If $\alpha>\beta, w>\beta$ or if $\alpha<\gamma, w<\gamma$, we have the corresponding solution is

$$
\pm\left(\xi-\xi_{0}\right)=\frac{1}{\sqrt{(\alpha-\beta)(\alpha-\gamma)}} \ln \frac{[\sqrt{(w-\beta)(\alpha-\gamma)}-\sqrt{(\alpha-\beta)(w-\gamma)}]^{2}}{|w-\alpha|} .
$$

The corresponding solution is 


$$
\begin{aligned}
& \pm\left(-\frac{1}{2} a_{3}\right)^{\frac{1}{4}}\left(\xi_{1}-\xi_{0}\right) \\
& =\frac{1}{\sqrt{(\alpha-\beta)(\alpha-\gamma)}} \ln \frac{\left\{\left(-\frac{1}{2} a_{3}\right)^{\frac{1}{4}}\left(u+\frac{a_{2}}{3 a_{3}}\right)-\beta\right](\alpha-\gamma)-\sqrt{(\alpha-\beta)\left[\left(-\frac{1}{2} a_{3}\right)^{\frac{1}{4}}\left(u+\frac{a_{2}}{3 a_{3}}\right)-\gamma \mid\right\}^{2}}}{\left|\left(-\frac{1}{2} a_{3}\right)^{\frac{1}{4}}\left(u+\frac{a_{2}}{3 a_{3}}\right)-\alpha\right|} .
\end{aligned}
$$

(ii) If $\alpha>\beta, w<\gamma$ or if $\alpha<\gamma, w<\beta$, we have

$$
\pm\left(\xi-\xi_{0}\right)=\frac{1}{\sqrt{(\alpha-\beta)(\alpha-\gamma)}} \ln \frac{[\sqrt{(w-\beta)(\gamma-\alpha)}-\sqrt{(\beta-\alpha)(w-\gamma)}]^{2}}{|w-\alpha|} .
$$

The corresponding solution is

$$
\begin{aligned}
& \pm\left(-\frac{1}{2} a_{3}\right)^{\frac{1}{4}}\left(\xi_{1}-\xi_{0}\right) \\
& =\frac{1}{\sqrt{(\alpha-\beta)(\alpha-\gamma)}} \ln \frac{\left\{\sqrt{\left[\left(-\frac{1}{2} a_{3}\right)^{\frac{1}{4}}\left(u+\frac{a_{2}}{3 a_{3}}\right)-\beta\right](\gamma-\alpha)}-\sqrt{\left.(\beta-\alpha)\left[\left(-\frac{1}{2} a_{3}\right)^{\frac{1}{4}}\left(u+\frac{a_{2}}{3 a_{3}}\right)-\gamma\right]\right\}^{2}}\right.}{\left|\left(-\frac{1}{2} a_{3}\right)^{\frac{1}{4}}\left(u+\frac{a_{2}}{3 a_{3}}\right)-\alpha\right|} .
\end{aligned}
$$

(iii) If $\beta>\alpha>\gamma$, we have

$$
\pm\left(\xi-\xi_{0}\right)=\frac{1}{\sqrt{(\beta-\alpha)(\alpha-\gamma)}} \arcsin \frac{(w-\beta)(\alpha-\gamma)+(\alpha-\beta)(w-\gamma)}{|(w-\alpha)(\beta-\gamma)|} .
$$

The corresponding solution is

$$
\begin{aligned}
& \pm\left(-\frac{1}{2} a_{3}\right)^{\frac{1}{4}}\left(\xi_{1}-\xi_{0}\right) \\
& =\frac{1}{\sqrt{(\beta-\alpha)(\alpha-\gamma)}} \arcsin \frac{\left[\left(-\frac{1}{2} a_{3}\right)^{\frac{1}{4}}\left(u+\frac{a_{2}}{3 a_{3}}\right)-\beta\right](\alpha-\gamma)+(\alpha-\beta)\left[\left(-\frac{1}{2} a_{3}\right)^{\frac{1}{4}}\left(u+\frac{a_{2}}{3 a_{3}}\right)-\gamma\right]}{\left|\left[\left(-\frac{1}{2} a_{3}\right)^{\frac{1}{4}}\left(u+\frac{a_{2}}{3 a_{3}}\right)-\alpha\right](\beta-\gamma)\right|} .
\end{aligned}
$$

Case 5. $D_{4}=0, D_{3}=0, D_{2}>0, E_{2}=0$. Then we have

$$
F(w)=(w-\alpha)^{3}(w-\beta) .
$$

where $\alpha$ and $\beta$ are real numbers.

When $\varepsilon=1$, if $w>\alpha, w>\beta$ or if $w<\alpha, w<\beta$, we have

$$
w=\frac{4(\alpha-\beta)}{(\alpha-\beta)^{2}\left(\xi-\xi_{0}\right)^{2}-4} \text {. }
$$


The corresponding solution is

$$
u=\left(\frac{1}{2} a_{3}\right)^{-\frac{1}{4}} \frac{4(\alpha-\beta)}{(\alpha-\beta)^{2}\left(\frac{1}{2} a_{3}\right)^{\frac{1}{2}}\left(\xi_{1}-\xi_{0}\right)^{2}-4}-\frac{a_{2}}{3 a_{3}} .
$$

When $\varepsilon=-1$, if $w>\alpha, w<\beta$ or if $w<\alpha, w>\beta$, we have

$$
w=\frac{4(\beta-\alpha)}{(\alpha-\beta)^{2}\left(\xi-\xi_{0}\right)^{2}+4} .
$$

The corresponding solution is

$$
u=\left(-\frac{1}{2} a_{3}\right)^{-\frac{1}{4}} \frac{4(\beta-\alpha)}{(\alpha-\beta)^{2}\left(-\frac{1}{2} a_{3}\right)^{\frac{1}{2}}\left(\xi_{1}-\xi_{0}\right)^{2}+4}-\frac{a_{2}}{3 a_{3}} .
$$

Case 6. $D_{4}=0, D_{2} D_{3}<0$. Then we have

$$
F(w)=(w-\alpha)^{2}\left[\left(w-l_{1}\right)^{2}+s_{1}^{2}\right] .
$$

where $\alpha, l_{1}$ and $s_{1}$ are real numbers.

When $\varepsilon=1$, we have

$$
w=\frac{\exp \left[ \pm \sqrt{\left(\alpha-l_{1}\right)^{2}+s_{1}^{2}}\left(\xi-\xi_{0}\right)\right]-\gamma+\sqrt{\left(\alpha-l_{1}\right)^{2}+s_{1}^{2}}}{\left\{\exp \left[ \pm \sqrt{\left(\alpha-l_{1}\right)^{2}+s_{1}^{2}}\left(\xi-\xi_{0}\right)\right]-\gamma\right\}^{2}-1} .
$$

The corresponding solution is

$$
u=\left(\frac{1}{2} a_{3}\right)^{-\frac{1}{4}} \frac{\exp \left[ \pm \sqrt{\left(\alpha-l_{1}\right)^{2}+s_{1}^{2}}\left(\frac{1}{2} a_{3}\right)^{\frac{1}{4}}\left(\xi_{1}-\xi_{0}\right)\right]-\gamma+\sqrt{\left(\alpha-l_{1}\right)^{2}+s_{1}^{2}}}{\left\{\exp \left[ \pm \sqrt{\left(\alpha-l_{1}\right)^{2}+s_{1}^{2}}\left(\frac{1}{2} a_{3}\right)^{\frac{1}{4}}\left(\xi_{1}-\xi_{0}\right)\right]-\gamma\right\}^{2}-1}-\frac{a_{2}}{3 a_{3}} .
$$

where $\gamma=\frac{\alpha-2 l_{1}}{\sqrt{\left(\alpha-l_{1}\right)^{2}+s_{1}^{2}}}$.

Case 7. $D_{4}>0, D_{3}>0, D_{1}>0$. Then we have

$$
F(w)=\left(w-\alpha_{1}\right)\left(w-\alpha_{2}\right)\left(w-\alpha_{3}\right)\left(w-\alpha_{4}\right) .
$$

where $\alpha_{1}, \alpha_{2}, \alpha_{3}$ and $\alpha_{4}$ are real numbers, and $\alpha_{1}>\alpha_{2}>\alpha_{3}>\alpha_{4}$.

When $\varepsilon=1$

(i) If $w>\alpha_{1}$ or $w<\alpha_{4}$, we have

$$
w=\frac{\alpha_{2}\left(\alpha_{1}-\alpha_{4}\right) \operatorname{sn}^{2}\left(\frac{\sqrt{\left(\alpha_{1}-\alpha_{3}\right)\left(\alpha_{2}-\alpha_{4}\right)}}{2}\left(\xi-\xi_{0}\right), m\right)-\alpha_{1}\left(\alpha_{2}-\alpha_{4}\right)}{\left(\alpha_{1}-\alpha_{4}\right) \operatorname{sn}^{2}\left(\frac{\sqrt{\left(\alpha_{1}-\alpha_{3}\right)\left(\alpha_{2}-\alpha_{4}\right)}}{2}\left(\xi-\xi_{0}\right), m\right)-\left(\alpha_{2}-\alpha_{4}\right)} .
$$


The corresponding solution is

$$
u=\left(\frac{1}{2} a_{3}\right)^{-\frac{1}{4}} \frac{\alpha_{2}\left(\alpha_{1}-\alpha_{4}\right) \operatorname{sn}^{2}\left(\frac{\sqrt{\left(\alpha_{1}-\alpha_{3}\right)\left(\alpha_{2}-\alpha_{4}\right)}}{2}\left(\frac{1}{2} a_{3}\right)^{\frac{1}{4}}\left(\xi_{1}-\xi_{0}\right), m\right)-\alpha_{1}\left(\alpha_{2}-\alpha_{4}\right)}{\left(\alpha_{1}-\alpha_{4}\right) \operatorname{sn}^{2}\left(\frac{\sqrt{\left(\alpha_{1}-\alpha_{3}\right)\left(\alpha_{2}-\alpha_{4}\right)}}{2}\left(\frac{1}{2} a_{3}\right)^{\frac{1}{4}}\left(\xi_{1}-\xi_{0}\right), m\right)-\left(\alpha_{2}-\alpha_{4}\right)}-\frac{a_{2}}{3 a_{3}} .
$$

(ii) If $\alpha_{2}>w>\alpha_{3}$, we have

$$
w=\frac{\alpha_{4}\left(\alpha_{2}-\alpha_{3}\right) \operatorname{sn}^{2}\left(\frac{\sqrt{\left(\alpha_{1}-\alpha_{3}\right)\left(\alpha_{2}-\alpha_{4}\right)}}{2}\left(\xi-\xi_{0}\right), m\right)-\alpha_{3}\left(\alpha_{2}-\alpha_{4}\right)}{\left(\alpha_{2}-\alpha_{3}\right) \operatorname{sn}^{2}\left(\frac{\sqrt{\left(\alpha_{1}-\alpha_{3}\right)\left(\alpha_{2}-\alpha_{4}\right)}}{2}\left(\xi-\xi_{0}\right), m\right)-\left(\alpha_{2}-\alpha_{4}\right)} .
$$

The corresponding solution is

$$
u=\left(\frac{1}{2} a_{3}\right)^{-\frac{1}{4}} \frac{\alpha_{4}\left(\alpha_{2}-\alpha_{3}\right) \operatorname{sn}^{2}\left(\frac{\sqrt{\left(\alpha_{1}-\alpha_{3}\right)\left(\alpha_{2}-\alpha_{4}\right)}}{2}\left(\frac{1}{2} a_{3}\right)^{\frac{1}{4}}\left(\xi_{1}-\xi_{0}\right), m\right)-\alpha_{3}\left(\alpha_{2}-\alpha_{4}\right)}{\left(\alpha_{2}-\alpha_{3}\right) \operatorname{sn}^{2}\left(\frac{\sqrt{\left(\alpha_{1}-\alpha_{3}\right)\left(\alpha_{2}-\alpha_{4}\right)}}{2}\left(\frac{1}{2} a_{3}\right)^{\frac{1}{4}}\left(\xi_{1}-\xi_{0}\right), m\right)-\left(\alpha_{2}-\alpha_{4}\right)}-\frac{a_{2}}{3 a_{3}} .
$$

When $\varepsilon=-1$

(i) $\alpha_{1}>w>\alpha_{2}$, we have

$$
w=\frac{\alpha_{3}\left(\alpha_{1}-\alpha_{2}\right) \operatorname{sn}^{2}\left(\frac{\sqrt{\left(\alpha_{1}-\alpha_{3}\right)\left(\alpha_{2}-\alpha_{4}\right)}}{2}\left(\xi-\xi_{0}\right), m\right)-\alpha_{2}\left(\alpha_{1}-\alpha_{3}\right)}{\left(\alpha_{1}-\alpha_{2}\right) \operatorname{sn}^{2}\left(\frac{\sqrt{\left(\alpha_{1}-\alpha_{3}\right)\left(\alpha_{2}-\alpha_{4}\right)}}{2}\left(\xi-\xi_{0}\right), m\right)-\left(\alpha_{1}-\alpha_{3}\right)} .
$$

The corresponding solution is

$$
u=\left(-\frac{1}{2} a_{3}\right)^{-\frac{1}{4}} \frac{\alpha_{3}\left(\alpha_{1}-\alpha_{2}\right) \operatorname{sn}^{2}\left(\frac{\sqrt{\left(\alpha_{1}-\alpha_{3}\right)\left(\alpha_{2}-\alpha_{4}\right)}}{2}\left(-\frac{1}{2} a_{3}\right)^{\frac{1}{4}}\left(\xi_{1}-\xi_{0}\right), m\right)-\alpha_{2}\left(\alpha_{1}-\alpha_{3}\right)}{\left(\alpha_{1}-\alpha_{2}\right) \operatorname{sn}^{2}\left(\frac{\sqrt{\left(\alpha_{1}-\alpha_{3}\right)\left(\alpha_{2}-\alpha_{4}\right)}}{2}\left(-\frac{1}{2} a_{3}\right)^{\frac{1}{4}}\left(\xi_{1}-\xi_{0}\right), m\right)-\left(\alpha_{1}-\alpha_{3}\right)}-\frac{a_{2}}{3 a_{3}} .
$$

(ii) $\alpha_{3}>w>\alpha_{4}$, we have

$$
w=\frac{\alpha_{1}\left(\alpha_{3}-\alpha_{4}\right) \operatorname{sn}^{2}\left(\frac{\sqrt{\left(\alpha_{1}-\alpha_{3}\right)\left(\alpha_{2}-\alpha_{4}\right)}}{2}\left(\xi-\xi_{0}\right), m\right)-\alpha_{4}\left(\alpha_{1}-\alpha_{3}\right)}{\left(\alpha_{3}-\alpha_{4}\right) \operatorname{sn}^{2}\left(\frac{\sqrt{\left(\alpha_{1}-\alpha_{3}\right)\left(\alpha_{2}-\alpha_{4}\right)}}{2}\left(\xi-\xi_{0}\right), m\right)-\left(\alpha_{3}-\alpha_{1}\right)} .
$$

The corresponding solution is 


$$
u=\left(-\frac{1}{2} a_{3}\right)^{-\frac{1}{4}} \frac{\alpha_{1}\left(\alpha_{3}-\alpha_{4}\right) \operatorname{sn}^{2}\left(\frac{\sqrt{\left(\alpha_{1}-\alpha_{3}\right)\left(\alpha_{2}-\alpha_{4}\right)}}{2}\left(-\frac{1}{2} a_{3}\right)^{\frac{1}{4}}\left(\xi_{1}-\xi_{0}\right), m\right)-\alpha_{4}\left(\alpha_{3}-\alpha_{1}\right)}{\left(\alpha_{3}-\alpha_{4}\right) \operatorname{sn}^{2}\left(\frac{\sqrt{\left(\alpha_{1}-\alpha_{3}\right)\left(\alpha_{2}-\alpha_{4}\right)}}{2}\left(-\frac{1}{2} a_{3}\right)^{\frac{1}{4}}\left(\xi_{1}-\xi_{0}\right), m\right)-\left(\alpha_{3}-\alpha_{1}\right)}-\frac{a_{2}}{3 a_{3}}
$$

where $m^{2}=\frac{\left(\alpha_{1}-\alpha_{2}\right)\left(\alpha_{3}-\alpha_{4}\right)}{\left(\alpha_{1}-\alpha_{3}\right)\left(\alpha_{2}-\alpha_{4}\right)}$.

Case 8. $D_{4}<0, D_{2} D_{3} \geq 0$. Then we have

$$
F(w)=(w-\alpha)(w-\beta)\left[\left(w-l_{1}\right)^{2}+s_{1}^{2}\right] .
$$

where $\alpha, \beta, l_{1}, s_{1}$ are real numbers, and $\alpha>\beta, s_{1}>0$.

When $\varepsilon=1$, we have

$$
w=\frac{a c n\left(\frac{\sqrt{-2 s_{1} m_{1}(\alpha-\beta)}}{2 m m_{1}}\left(\xi-\xi_{0}\right), m\right)+b}{\operatorname{ccn}\left(\frac{\sqrt{-2 s_{1} m_{1}(\alpha-\beta)}}{2 m m_{1}}\left(\xi-\xi_{0}\right), m\right)+d} .
$$

The corresponding solution is

$$
u=\left(\frac{1}{2} a_{3}\right)^{-\frac{1}{4}} \frac{a \operatorname{cn}\left(\frac{\sqrt{-2 s_{1} m_{1}(\alpha-\beta)}}{2 m m_{1}}\left(\frac{1}{2} a_{3}\right)^{\frac{1}{4}}\left(\xi_{1}-\xi_{0}\right), m\right)+b}{\operatorname{ccn}\left(\frac{\sqrt{-2 s_{1} m_{1}(\alpha-\beta)}}{2 m m_{1}}\left(\frac{1}{2} a_{3}\right)^{\frac{1}{4}}\left(\xi_{1}-\xi_{0}\right), m\right)+d}-\frac{a_{2}}{3 a_{3}} .
$$

When $\varepsilon=-1$, we have

$$
w=\frac{a c n\left(\frac{\sqrt{2 s_{1} m_{1}(\alpha-\beta)}}{2 m m_{1}}\left(\xi-\xi_{0}\right), m\right)+b}{\operatorname{ccn}\left(\frac{\sqrt{2 s_{1} m_{1}(\alpha-\beta)}}{2 m m_{1}}\left(\xi-\xi_{0}\right), m\right)+d} .
$$

The corresponding solution is

$$
u=\left(-\frac{1}{2} a_{3}\right)^{-\frac{1}{4}} \frac{a \operatorname{cn}\left(\frac{\sqrt{2 s_{1} m_{1}(\alpha-\beta)}}{2 m m_{1}}\left(-\frac{1}{2} a_{3}\right)^{\frac{1}{4}}\left(\xi_{1}-\xi_{0}\right), m\right)+b}{\operatorname{ccn}\left(\frac{\sqrt{2 s_{1} m_{1}(\alpha-\beta)}}{2 m m_{1}}\left(-\frac{1}{2} a_{3}\right)^{\frac{1}{4}}\left(\xi_{1}-\xi_{0}\right), m\right)+d}-\frac{a_{2}}{3 a_{3}} .
$$

where

$$
\begin{aligned}
& c=a-l_{1}-\frac{s_{1}}{m_{1}}, d=a-l_{1}-s_{1} m_{1}, a=\frac{1}{2}[(\alpha+\beta) c-(\alpha-\beta) d], \\
& b=\frac{1}{2}[(\alpha+\beta) d-(\alpha-\beta) c], E=\frac{s_{1}^{2}+\left(\alpha-l_{1}\right)\left(\beta-l_{1}\right)}{s_{1}(\alpha-\beta)}, \\
& m_{1}=E \pm \sqrt{E^{2}+1}, m^{2}=\frac{1}{1+m_{1}^{2}} .
\end{aligned}
$$


We choose $m_{1}$ such that $\varepsilon m_{1}<0$.

Case 9. $D_{4}>0, D_{2} D_{3} \leq 0$. Then we have

$$
F(w)=\left[\left(w-l_{1}\right)^{2}+s_{1}^{2}\right]\left[\left(w-l_{2}\right)^{2}+s_{2}^{2}\right] .
$$

where $l_{1}, l_{2}, s_{1}, s_{2}$ are real numbers, and $s_{1}>s_{2}>0$.

When $\varepsilon=1$, we have

$$
w=\frac{\operatorname{asn}\left(\eta\left(\xi-\xi_{0}\right), m\right)+b \operatorname{cn}\left(\eta\left(\xi-\xi_{0}\right), m\right)}{\operatorname{csn}\left(\eta\left(\xi-\xi_{0}\right), m\right)+d \operatorname{cn}\left(\eta\left(\xi-\xi_{0}\right), m\right)} .
$$

The corresponding solution is

$$
u=\left(\frac{1}{2} a_{3}\right)^{-\frac{1}{4}} \frac{\operatorname{asn}\left(\eta\left(\frac{1}{2} a_{3}\right)^{\frac{1}{4}}\left(\xi_{1}-\xi_{0}\right), m\right)+b \operatorname{cn}\left(\eta\left(\frac{1}{2} a_{3}\right)^{\frac{1}{4}}\left(\xi_{1}-\xi_{0}\right), m\right)}{\operatorname{csn}\left(\eta\left(\frac{1}{2} a_{3}\right)^{\frac{1}{4}}\left(\xi_{1}-\xi_{0}\right), m\right)+d \operatorname{cn}\left(\eta\left(\frac{1}{2} a_{3}\right)^{\frac{1}{4}}\left(\xi_{1}-\xi_{0}\right), m\right)}-\frac{a_{2}}{3 a_{3}} .
$$

where

$$
\begin{aligned}
& c=-s_{1}-\frac{s_{2}}{m_{1}}, d=l_{1}-l_{2}, a=l_{1} c+s_{1} d, b=l_{1} d-s_{1} c, \\
& E=\frac{s_{1}^{2}+s_{2}^{2}+\left(l_{1}-l_{2}\right)^{2}}{2 s_{1} s_{2}}, m_{1}=E+\sqrt{E^{2}-1}, m^{2}=1-\frac{1}{m_{1}^{2}}, \eta=s_{2} \sqrt{\frac{m_{1}^{2} c^{2}+d^{2}}{c^{2}+d^{2}}} .
\end{aligned}
$$

In Equations (21) (24) (27) (29) (32) (34) (36) (38) (40) (42) (45) (47) (50) (53) (55) (57) (59) (62) (64) and (68), the integration constant $\xi_{0}$ has been rewritten, but we still use it. The classifications of all single traveling wave solution to this equation are obtained.

\section{Conclusion}

In this paper, the trial equation method combined with complete discrimination system for polynomial has been effectively used to solve the Getmanou equation. The obtained results emphasize that the method is completely useful. With the same method, some of other equations can be dealt with.

\section{Acknowledgements}

I would like to thank the referees for their valuable suggestions.

\section{References}

[1] Ablowitz, M.J. and Clarkson, P.A. (1992) Solitons, Nonlinear Evolution Equations and Inverse Scattering. Cambridge University Press, Cambridge, 721-725.

[2] Satsuma, J. and Kaup D.J. (1977) A Bäcklund Transformation for a Higher Order Korteweg-De Vries Equation. Journal of the Physical Society of Japan, 43, 692-697. http://dx.doi.org/10.1143/JPSJ.43.692

[3] Gu, C., Hu, H. and Zhou, Z. (2006) Darboux Transformations in Integrable Systems: Theory and Their Applications to Geometry. Springer, Berlin, Heidelberg.

[4] Wang, M., Zhou, Y. and Li, Z. (1996) Application of a Homogeneous Balance Method to Exact Solutions of Nonlinear Equations in Mathematical Physics. Physics Letters A, 216, 67-75. http://dx.doi.org/10.1016/0375-9601(96)00283-6

[5] Fan, E. (2000) Extended tanh-Function Method and Its Applications to Nonlinear Equations. Physics Letters A, 277, 212-218. http://dx.doi.org/10.1016/S0375-9601(00)00725-8

[6] He, J.H. and Zhang, L.N. (2008) Generalized Solitary Solution and Compacton-Like Solution of the Jaulent-Miodek Equations Using the Exp-Function Method. Physics Letters A, 372, 1044-1047. http://dx.doi.org/10.1016/j.physleta.2007.08.059 
[7] Wang, M., Li, X. and Zhang, J. (2008) The (G'/G)-Expansion Method and Travelling Wave Solutions of Nonlinear Evolution Equations in Mathematical Physics. Physics Letters A, 372, 417-423. http://dx.doi.org/10.1016/j.physleta.2007.07.051

[8] Liu, C.S. (2006) Trial Equation Method to Nonlinear Evolution Equations with Rank Inhomogenous: Mathematical Discussions and Its Applications. Communications in Theoretical Physics, 45, 219-223. http://dx.doi.org/10.1088/0253-6102/45/2/005

[9] Liu, C.S. (2005) Trial Equation Method and Its Applications to Nonlinear Evolution Equations. Acta Physica Sinica, 54, 2505. (in Chinese)

[10] Liu, C.S. (2007) Classification of All Single Travelling Wave Solutions to Calogero-Degasperis-Focas Equation. Communications in Theoretical Physics, 48, 601-604. http://dx.doi.org/10.1088/0253-6102/48/4/004

[11] Liu, C.S. (2006) Direct Integral Method, Complete Discrimination System for Polynomial and Applications to Classifications of All Single Traveling Wave Solutions to Nonlinear Differential Equations: A Survey. arXiv preprint nlin/ 0609058.

[12] Liu, C.S. (2008) Solution of ODE $u^{\prime \prime}+p(u)\left(u^{\prime}\right)^{2}+q(u)=0$ and Applications to Classifications of All Single Travelling Wave Solutions to Some Nonlinear Mathematical Physics Equations. Communications in Theoretical Physics, 49, 291296. http://dx.doi.org/10.1088/0253-6102/49/2/07

[13] Fan, X.-F. (2010) Classification of All Single Traveling-Wave Solutions to Getmanou Equation. FIZIKA A, 19, 191196. 